\title{
Innovazioni negli accessi vascolari
}

\author{
Mario Cifarelli, Paolo Altamura
}

\author{
U.O. di Chirurgia Vascolare \\ Azienda Ospedaliera "Di Venere - Giovanni XXIII", Bari - Carbonara
}

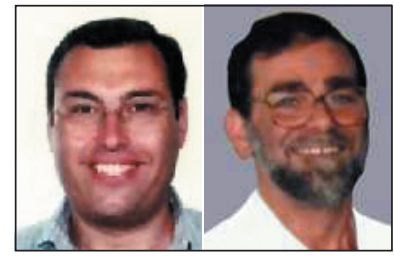

Mario Cifarelli e Paolo Altamura Brescia,non sono state più fatte scoperte così innovative da determinare simili rivoluzioni epocali, tuttavia la ricerca scientifica ha costantemente lavorato per incrementare la durata e migliorare la sicurezza degli accessi vascolari, che costituiscono uno dei problemi più sentiti dal paziente emodializzato e dal nefrologo e che sono tuttora una delle principali cause di ospedalizzazione.

Negli ultimi anni, gli sforzi dei ricercatori e delle industrie si sono indirizzati soprattutto verso tre campi di applicazione ottenendo risultati soddisfacenti e anche abbastanza promettenti: l'angioradiologia interventistica, i materiali protesici, i cateteri venosi centrali.

Ben lungi dal risolvere il problema, si è riusciti, tuttavia, a incrementare sempre più la durata degli accessi vascolari riducendo significativamente, soprattutto, l'incidenza delle temibili complicanze a essi connesse. L'incremento della sopravvivenza media del paziente emodializzato si deve anche a questi successi parziali, che incoraggiano a proseguire sulle strade intraprese.

Vedremo ora di analizzare in dettaglio i risultati fino a oggi raggiunti e le prospettive future.

\section{Angioradiologia interventistica}

Dalle prime angioplastiche con cateteri a palloncino e dopo i primi esaltanti successi in campo cardiologico $\mathrm{e}$, successivamente, angiologico degli anni ' 80 , si sono sempre più sviluppati metodiche e devices che potessero risolvere stenosi e ostruzioni altrimenti non recuperabili se non con un intervento chirurgico. In tempi più recenti, questo bagaglio tecnico è stato trasferito anche nel campo degli accessi vascolari ottenendo egualmente lusinghieri successi grazie, anche, a una più accurata sorveglianza, che ha consentito interventi precoci.

Attualmente, $i$ settori in cui ci possiamo avvalere dell' angioradiologia sono la diagnostica fine (angiografia), il trattamento delle stenosi (PTA e stenting), la trombolisi (farmacomeccanica e meccanica).

L'angiografia consente la precisa localizzazione di stenosi, particolarmente nelle sedi difficilmente indagabili dagli ultrasuoni (vene anonime, succlavie, iliache, cave), consentendo una valutazione abbastanza precisa del grado di restringimento e della situazione globale dell'accesso. In tempi recenti, è stato introdotto come mezzo di contrasto il diossido di carbonio per sostituire gli agenti iodati, che, soprattutto nel dializzato, comportano un certo grado di rischio, ottenendo delle immagini di qualità appena inferiore.

I cateteri a palloncino attuali sono sempre più sofisticati e sono in grado di sopportare anche pressioni di gonfiaggio superiori a 25 atmosfere (Blue-Max, Medi-tech $\%$ Centurion, $\operatorname{Bard}^{\circ}$ ) capaci di superare le stenosi degli accessi vascolari che sono notoriamente molto resistenti. Recentemente, per stenosi ancora più rigide, sono stati messi a punto dei palloncini taglienti anche se disponibili solo per calibri inferiori ai $4 \mathrm{~mm}$ (1). Perfino occlusioni complete, se di brevi seg- 
menti, possono essere superate da fili guida e, successivamente, dilatate. Le indicazioni alla PTA si sono estese alla maggior parte delle stenosi degli accessi vascolari con particolare successo nel trattamento di quelle da iperplasia intimale a livello delle anastomosi protesico-venose e in quelle venose succlavie, causa di edema dell'arto (sindrome del "gros bras"). Poche, invece, le controindicazioni: infezioni locali, stenosi anastomotiche di FAV recenti, stenosi isolate a meno di $10 \mathrm{~cm}$ dal polso nelle Cimino-Brescia, stenosi lunghe e occlusioni croniche.

I risultati ottenuti dalla PTA sono stati migliorati dall'introduzione dello stenting, che ha ridotto le percentuali di restenosi e risolto una gran parte delle complicanze legate alla procedura. Dopo i primi stent rigidi, sono stati applicati prodotti sempre più sofisticati con architetture e materiali capaci di autoespandersi (Nitinol), di essere posizionati a livello delle pieghe articolari per la loro flessibilità (Wallstent, Craggstent), di riparare aneurismi, pseudoaneurismi e lacerazioni vasali grazie al loro rivestimento (covered stent). La pervietà a distanza, soprattutto secondaria, raggiunge ottime percentuali fino a oltre il $90 \%$ ad 1 anno e 1' $80 \%$ a 2 anni in alcune casistiche (2).

Verso la fine degli anni Ottanta, ha cominciato a diffondersi la pratica della trombolisi farmacomeccanica (PMT) per disostruire accessi, in particolare protesici, trombizzati da poco tempo. L'osservazione che la semplice trombolisi farmacologica con urochinasi era gravata da frequenti insuccessi e da complicanze sistemiche piuttosto gravi, indusse numerosi ricercatori a utilizzare sistemi locali più efficaci e meno rischiosi. La metodica pulse-spray ha centrato tale obiettivo grazie all'uso di particolari cateteri (Angiodynamics Inc..$^{\odot}$ ) provvisti di numerosi fori ravvicinati, che si aprono solo sotto pressione, quando viene iniettato l'agente litico, determinando l'effetto spray ed evitando l'entrata del sangue. Piccoli volumi successivi di urochinasi $(0.2-0.4 \mathrm{ml})$ sono in grado di lisare il trombo spingendo pro- gressivamente il catetere verso le estremità venosa e arteriosa del graft. La procedura combinata con l'angiografia e la PTA successiva ha permesso un alto tasso di successo $96 \%$ con solo il 3\% di complicanze minori (3). Successivamente, Beathard et al (4) hanno dimostrato che la sola azione meccanica del pulse-spray era già sufficiente a frammentare il trombo e, utilizzando una tecnica combinata di spray con semplice soluzione salina e tromboaspirazione, ottennero il $100 \%$ di risultati positivi entro le 48 ore evitando l'uso di agenti trombolitici.

La più recente innovazione in questo campo è costituita dalla trombolisi meccanica, che si avvale di apparecchiature di rimozione del trombo tramite sistemi a ricircolo, rotazionali o idrodinamici (Amplatz, Microvena ${ }^{\odot}$ Hydrolyzer, $\mathrm{Cordis}^{\odot}$ ) (5) e meccanici (Trerotola, Arrow Int. ${ }^{\circ}$ ) (6). Questi strumenti, grazie all'impiego di microturbine, che creano dei vortici idrodinamici o azionano cestelli metallici, sono in grado di frammentare il trombo e contemporaneamente di aspirarne i residui. Le percentuali di successo sono più che soddisfacenti (oltre il 90\%).

\section{Protesi}

L'accesso vascolare protesico è l'accesso più diffuso nelle Scuole nordamericane e spesso viene eseguito perfino di prima istanza. Considerando l'alta incidenza di complicanze connesse, oltre alla ridotta sopravvivenza nel tempo, la ricerca e soprattutto le industrie di quei Paesi hanno indirizzato i loro sforzi per migliorare e innovare i materiali cercando di avvicinarli quanto più possibile alle caratteristiche ideali.

Il materiale di scelta è stato per anni il PTFE, nonostante le non esaltanti performances alla distanza e, per tale motivo, è stato quello su cui maggiormente si è lavorato. Inizialmente si è cercato di ridurre la trombogenicità rivestendo la superficie interna con il carbone pirolitico $\left(\right.$ Carboflo, IMPRA ${ }^{\circ}$ ) sulla scorta delle esperienze maturate nel campo delle valvole meccaniche cardiache. Con un processo di fabbri- cazione denominato "coestrusione" si è ottenuto una parete protesica monostrato di PTFE e carbonio. L'impiego di piastrine marcate ha evidenziato una marcata riduzione della loro adesione parietale (7) e, quindi, della trombogenicità, tuttavia l'esperienza clinica non ha mostrato significative differenze rispetto al PTFE standard. Infatti, la causa principale della trombosi protesica è da ricercare soprattutto nella iperplasia intimale a livello dell'anastomosi venosa, determinata, a sua volta, dal compliance mismatch tra la parete del graft e quella vasale. Per cercare di attenuare questo inconveniente, i ricercatori hanno messo a punto protesi a parete sottile (thinwall, $\mathrm{IMPRA}^{\circ}$ ) e cercato di migliorare il profilo emodinamico progettando architetture coniche. Negli ultimi anni, sulla scorta di ricerche svolte da Scholz (8), a loro volta riprese da una intuizione di Miller, sono state proposte delle protesi in PTFE con l'estremità a cuffia da anastomizzare al vaso venoso (Venaflo, IMPRA ${ }^{\oplus}$ ). Questa configurazione a due pezzi, patch di ampliamento e protesi, ha mostrato, in un modello sperimentale ovino, una riduzione del $50 \%$ dell'iperplasia intimale.

Una delle complicanze più gravi e purtroppo frequenti che possono insorgere in un accesso protesico è costituita dalla lacerazione parietale con formazione di pseudoaneurismi. Per rendere più resistente il PTFE al trauma continuo provocato dagli aghi da dialisi, i bioingegneri hanno prodotto una protesi con parete rinforzata costituita da più strati sovrapposti di fibrille di PTFE variamente orientate, in grado di chiudere ermeticamente $\mathrm{i}$ fori degli aghi (Diastat, Gore-Tex ${ }^{\odot}$ ). I risultati clinici hanno dato risultati soddisfacenti per quanto riguarda la riduzione della complicanza, ma non così per la pervietà a distanza.

Rimanendo nel campo delle protesi sintetiche, mentre tutti gli altri materiali sono stati pressoché abbandonati, promettente risulta un nuovo elastomero di policarbonato (Chrono Flex) con cui ultimamente sono fabbricati grafts (VascuLink, Credent ${ }^{\circledR}$ ) dalle caratteristiche interessanti: utilizzabilità 
precoce, buona compliance, maneggevolezza chirurgica, facile pungibilità, resistenza alla puntura, ridotto sanguinamento. Le premesse sono incoraggianti anche se le esperienze cliniche sono ancora limitate rispetto al PTFE.

La continua ricerca di materiali con caratteristiche il più compatibili possibili con i vasi nativi, ha indotto a sperimentare protesi biologiche di derivazione sia omologa che eterologa. Queste hanno dei grossi vantaggi rispetto alle sintetiche, in particolare quelli di avere una trombogenicità inferiore e, soprattutto, un minore compliance mismatch, anche se sono meno resistenti alle punture e alla formazione di aneurismi. Per tali motivi si è lavorato sia per migliorare i sistemi di conservazione, principale causa di biodegradabilità e perdita di resistenza parietale sia per fornire rinforzo esterno. Sono così nate le protesi biosintetiche, definite in tal modo per la presenza di una rete di Dacron esterna o insita nella parete stessa.

Le vene safene omologhe sia fresche che conservate sono state le prime a essere utilizzate, ma la non sempre immediata disponibilità di segmenti idonei, i rischi di elevata antigenicità e l'alta incidenza di aneurismi hanno ridotto notevolmente l'impiego di questa protesi, anche se alcuni autori (9) riportano soddisfacenti percentuali di pervietà con vene crioconservate. Anche la vena ombelicale umana (Dardik, Biograft ${ }^{\odot}$ ) rinforzata da mesh di Dacron e la carotide bovina, quest'ultima largamente usata in passato, hanno avuto un successo limitato per l'alta incidenza di aneurismi. Infatti, il trattamento denaturante proteico cui venivano sottoposte le rendeva dei condotti inerti.

Attualmente, le protesi di questo tipo che stanno dimostrando caratteristiche di durata e resistenza superiori sono due: la Omniflow, Bionova $^{\odot}$ e la Procol, Hancock ${ }^{\odot}$. La prima viene ottenuta inserendo nel sottocute di pecore vive un mandrino con una rete a maglie larghe di dacron, che provoca una reazione connettivale. È disponibile retta o precurvata per gli impianti a loop e ha una buona compliance con ridotti fenomeni di iperplasia miointimale e, nella sua nuova versione con rete a maglie più fitte (Omniflow II), risulta maggiormente resistente alle punture.

La seconda è ottenuta, con procedimento in parte segreto, dal prelievo e dal trattamento di vena di vitello (vena mesenterica) e dall'aggiunta di una rete di dacron esterna. Introdotta in commercio alcuni anni fa, nonostante i buoni risultati iniziali, non fu più prodotta fino alla fine degli anni '90, quando ne è ripresa la commercializzazione. Le caratteristiche di duttilità chirurgica, di resistenza alle infezioni, la relativamente bassa incidenza di aneurismi, la buona compliance e $\mathrm{i}$ primi risultati sulla pervietà alla distanza, ne incoraggiano la diffusione.

\section{Cateteri venosi centrali}

Anche se l'uso di cateteri venosi centrali (CVC) per l'emodialisi è stato proposto fin dal 1970 , la loro diffusione ed evoluzione tecnologica è piuttosto recente. Attualmente, sono da considerarsi l'accesso di scelta nelle dialisi acute e uno dei più utilizzati nelle croniche, con prospettive ulteriori di sviluppo. Per quanto presentino degli indubbi vantaggi (facilità di impianto e di sostituzione, precocità nell'utilizzo, non necessità di puntura), tuttavia essi sono soggetti a due complicanze importanti e, purtroppo, ancora frequenti: l'ostruzione/bassa portata e l'infezione. Quest'ultima risulta negli USA la principale causa di batteriemia nel dializzato con una incidenza di 3.9 episodi/1000 giorni/catetere (10). Gli sforzi innovativi, quindi, si sono indirizzati al tentativo di risolvere questi problemi, sperimentando nuovi materiali meno trombogeni e più resistenti, nuove conformazioni, nuovi sistemi di prevenzione delle infezioni.

L'ostruzione/bassa portata è determinata dalla formazione di una camicia di fibrina (fibrin sleeve) che incarcera i fori del CVC impedendo l'aspirazione del sangue. Per questo, si è cercato di utilizzare materiali quanto più possibile inerti e con superfici prive di irregolarità per prevenire l'adesione piastrinica e che, contemporaneamente, mantenessero le caratteristiche di resistenza, flessibilità e sottigliezza della parete. Ne sono stati sperimentati vari: polietilene, teflon, silicone, poliuretano, ma, attualmente, solo gli ultimi due vengono considerati rispondenti ai requisiti.

Il silicone è termoformato e si presenta morbido, ma richiede una parete più spessa per evitare kinkings; con esso sono costruiti i cateteri più diffusi: Permcath, Mahurkar (Quinton ${ }^{\odot}$ ), Vascath $\left(\operatorname{Bard}^{\odot}\right)$. Il poliuretano è termoplastico, per cui diventa morbido alla temperatura corporea; si presenta resistente, ma si deteriora facilmente a contatto con alcool. Recentemente sono stati impiegati dei polimeri del poliuretano per rivestire le pareti dei CVC creando una superficie estremamente liscia, in grado di ridurre le interazioni con proteine e piastrine (GamCath, Gambro ${ }^{\circledR}$ ).

Il problema delle infezioni è stato affrontato su più fronti. L'inserimento di una cuffia di teflon a manicotto ha ridotto la contaminazione esterna del CVC e dell'exit-site, grazie alla reazione cicatriziale provocata. Ben più pericolose, per il rischio di batteriemia, sono le infezioni della superficie interna, assai resistenti alle terapie antibiotiche. Interessanti studi di Stewart e Costerton (11) hanno recentemente dimostrato come le colonie batteriche creano un biofilm dal quale sono protette rispetto alle popolazioni planctoniche mostrando livelli di resistenza agli antibiotici fino a 1500 volte superiori. Per evitare la formazione del biofilm, si sta cercando di agire sia sui materiali di costruzione dei CVC sia sui liquidi di block. Il trattamento delle superfici con argento micronizzato mediante un procedimento di rivestimento particolare (ion beamassisted deposition) ha consentito di ridurre drasticamente le popolazioni batteriche ( $8 \%$ contro il $46.4 \%$ ) (12). L'uso di liquidi di block diversi dalla semplice eparina, come il citrato associato a taurolidina, sembra efficace nella prevenzione del biofilm. Ancora a livello sperimentale, ma piuttosto interessanti, sono la polygelina e l'AI-2 Furanone estratto dalla Delisea Pulchra. 
Il futuro dei CVC, però, sembra riposto nei sistemi totalmente impiantabili sia per la minore esposizione alle infezioni sia per l'aspetto cosmetico e il maggior grado di accettazione da parte dei pazienti. Attualmente, ne sono in commercio 2 tipi: il Dialock, Biolink ${ }^{\odot}$ e il LifeSite, Vasca $^{\circ}$, anche se l'esperienza clinica del secondo è ancora piuttosto limitata(13).

Derivati dalle esperienze maturate con i port, sono costituiti da due cateteri posizionati come i CVC classici e collegati con un dispositivo metallico sottocutaneo fornito di valvole, che viene punto attraverso la cute. Il Dialock, che già vanta numerosi impianti in Italia e un discreto follow-up, permette di ottenere flussi adeguati, senza le angolazioni della corrente ematica che impone, invece, il LifeSite, ma mantiene ancora dei costi molto alti. I vantaggi in termini di prevenzione dalle infezioni sono nettamente a favore dei sistemi totalmente impiantabili con una incidenza di infezioni di 0.78 episodi/ 1000 giorni/ catetere rispetto ai 3.9 dei CVC esterni. La conclusione di trials multicentrici già in corso fornirà sicuramente dati interessanti anche in termini di rapporto costo/beneficio.

p.altamura@tin.it

\section{BIBLIOGRAFIA}

1. Vorwerk D, Adam A, MuellerLeisse C, Guenther R. Hemodialysis fistulas and grafts: use of cutting balloons to dilate venous stenosis. Radiology 1996; 201: 8647.

2. Turmel-Rodrigues L, Blanchard D, Pengloan J, et al. Treatment of failing and failed hemodialysis fistulae and grafts by interventional radiology: long-term follow-up. 1999; in press. Nephrol Dial Transplant $2000 ; 15$ (12): 2009-36.

3. Valji K, Bookstein JJ, Roberts AC, et al. Pulse-spray pharmacomechanicalthrombolysis of thrombosed hemodialysis access grafts: long-term experience and comparison of original and current techniques. Am J Radiol 1995; 164: 1495-500.

4. Beathard GA, Welch BR, Maidment HJ. Mechanical thrombolysis for the treatment of thrombosed dialysis access grafts: report of a series. Radiology 1996; 200: 711-6.

5. Vorwerk D, Sohn M, Schurmann K, et al. Hydrodynamic thrombectomy of hemodialysis fistulas: first clinical results. J Vascular and Interventional Radiology 1994; 5: 813-21.

6. Trerotola SO, Vesely TM, Lund GB, et al. Treatment of thrombosed hemodialysis access grafts: ArrowTrerotola percutaneous thrombolytic device versus pulse-spray thrombolysis. Radiology 1998; 206: 403-14.

7. Tsuchida H, Cameron BL, Marcus CS, Wilson SE. Modified polytetrafluoroethylene: Indium 111-labeled platelet deposition on carbon-lined and high porosity polytetrafluoroethylene grafts. J Vascul Surg 1992; 4: 643-9.

8. Scholz H. Five years experience with arteriovenous patchpro- sthesis (AVP) as access for hemodialysis. The Sixth Bi-Annual Symposium on Dialysis Access, Miami 1998.

9. Berardinelli L, Vegeto A. Lessons from 494 permanent accesses in 348 haemodialysis patients older than 65 years of age: 29 years of experience. Nephrol Dial Transplant, 1998; 13 (Suppl): S73-7.

10. Marr KA, Sexton DJ, Conlon $\mathrm{PJ}$, et al. Catheter-related bacteriemia and outcome of attempted catheter salvage in patients undergoing hemodialysis. Ann Intern Med 1997; 127: 275-80.

11. Stewart PS, Costerton JW. Antibiotic resistance of bacteria in biofilms. Lancet 2001; 14: 135-8.

12. Bambauer R, Mestres P, Schiel JM, et al. Large bore catheters with surface treatments versus untreated catheters for blood access. The Journal of Vascular Access, 2001; 2: 97-105.

13. Beathard GA, Posen GA. Initial clinical results with the LifeSite Hemodialysis Access System. Kidney Int 2000; 58: 2221-7. 